\title{
Unraveling Resilience: Personality Predicts Exposure and Reaction to Stressful Life Events
}

\author{
Eva Asselmann ${ }^{1}$, Theo A. Klimstra ${ }^{2,3}$, Jaap J. A. Denissen ${ }^{4}$
}

[1] Faculty of Health, HMU Health and Medical University, Potsdam, Germany. [2] Department of Developmental Psychology, Tilburg School of Social and Behavioral Sciences, Tilburg University, Tilburg, the Netherlands. [3] EliotPearson Department of Child Study and Human Development, Tufts University, Medford, MA, USA. [4] Department of Developmental Psychology, Faculty of Social and Behavioural Sciences, Utrecht University, Utrecht, the Netherlands.

Personality Science, 2021, Vol. 2, Article e6055, https://doi.org/10.5964/ps.6055

Received: 2021-02-02 • Accepted: 2021-08-12 • Published (VoR): 2021-11-04

Handling Editor: Ryne A. Sherman, Hogan Assessment Systems, Tulsa, OK, USA

Reviewing: Round 1 - Josh Jackson; Anonymous \#1. No open reviews are available.

Corresponding Author: Eva Asselmann, HMU Health and Medical University, Faculty of Health, Olympischer Weg 1, 14471 Potsdam, Germany. Fax: 49331745113 131, E-mail: eva.asselmann@health-and-medical-university.de

Supplementary Materials: Data, Materials, Preregistration [see Index of Supplementary Materials]

\section{Abstract}

Resilience is a key construct in psychology, which describes the maintenance of comparatively good mental health despite of environmental adversities or successful recovery from such adversities. Furthermore, it labels a specific personality type, characterized by high levels across the Big Five. However, whether the resilient type predicts less unfavorable mental health changes around environmental adversities remains unresolved. In a nationally representative sample from the Netherlands (LISS panel, $\mathrm{N}=12,551$ ), we longitudinally examined whether changes of internalizing symptoms around four stressful life events (unemployment, disability, divorce, and widowhood) differed between resilients and non-resilients. Internalizing symptoms increased before but decreased after each event, indicating recovery. Compared to non-resilients, resilients experienced a weaker symptom increase before the onset of unemployment and a stronger symptom rebound after the onset of disability. Thus, resilients maintained higher levels of mental health and recovered faster when faced with specific adversities, which underscores the importance of personality types in resilience.

\section{Keywords}

Big Five, personality type, longitudinal, adjustment, mental health, psychopathology, internalizing, substance use 


\section{Relevance Statement}

Stressful life events occur in the life of many people. Here, we investigated how psychopathological symptoms change in the years before and after the onset of unemployment, disability, divorce, and widowhood. We also tested whether these changes differ between two major personality types: Resilients, who score comparatively high across the Big Five traits extraversion, agreeableness, conscientiousness, emotional stability, and openness versus non-resilients, who score lower on these traits. Compared to non-resilients, resilients experienced a weaker symptom increase before the onset of unemployment and recovered better after the onset of disability. These findings underscore that personality trait configurations can predict individual differences in people's ability to cope with adverse experiences. Assessing non-resilient personality types could improve an early identification of high-risk individuals, who might benefit from targeted prevention.

\section{Key Insights}

- We examined changes of internalizing symptoms around stressful life events.

- We tested whether these changes vary by personality type.

- We used data from a nationally representative sample from the Netherlands.

- Before unemployment, symptoms increased less in resilients versus non-resilients.

- Moreover, resilients recovered better after the onset of disability.

Stressful life events such as unemployment, divorce, and the death of a partner affect many people (Denissen et al., 2019). Although such experiences relate to an increase of psychopathological symptoms (Infurna \& Luthar, 2016, 2017; Kim \& von Dem Knesebeck, 2016; Vable et al., 2015) and are risk factors for mental disorders (Asselmann et al., 2016), some people show resilience when faced with environmental adversities (Galatzer-Levy et al., 2018). However, resilience can be seen from multiple perspectives. Combining these perspectives promises to enhance scientific integration and progress in the field.

From a first within-person perspective, resilience describes the phenomenon that some people maintain or regain good mental health despite substantial adversities (Luthar et al., 2000). From this point of view, resilience refers to the absence of mental health problems or to a comparatively small increase of and/or good recovery from psychopathological symptoms before and after stressful events. Thus, a resilient versus non-resilient within-person symptom trajectory would translate into relatively low-toaverage levels of psychopathological symptoms around adverse experiences.

From a second between-person perspective, resilience labels a specific personality type, originally introduced by Block (1971). Block distinguished three developmental types in childhood: Resilients (characterized by high ego-resilience and medium ego-control), undercontrollers (characterized by low ego-resilience and low ego-control), and overcontrollers (characterized by low ego-resilience and high ego-control; Asendorpf \& 
van Aken, 1999; Block, 1971; Chapman \& Goldberg, 2011; Denissen et al., 2008; Hart et al., 1997; Hart et al., 2003; Robins et al., 1996). In Block's and other studies, these developmental types in childhood predicted developmental outcomes up into adulthood in a way that resilients tended to experience more favorable outcomes than non-resilient types (e.g., higher levels of mental health, cognitive abilities, and psychosocial functioning; Asendorpf \& Denissen, 2006; Asendorpf \& van Aken, 1999; Caspi, 2000; Caspi et al., 1996; Caspi et al., 2003; Denissen et al., 2008; Newman et al., 1997). However, Block came up with these type labels (i.e., resilient and non-resilient) irrespective of within-person symptom changes around stressful events.

In adolescents and adults, similar types to those reported by Block have been distinguished based on the Big Five traits extraversion, agreeableness, conscientiousness, emotional stability, and openness (Asendorpf \& van Aken, 1999; Robins et al., 1996). In this research, resilients scored comparatively high across the Big Five, whereas non-resilients (i.e., under- and overcontrollers) scored lower on (some of) these traits. In adolescents and adults, cross-sectional and longitudinal studies also revealed that the resilient type (based on the Big Five) was associated with more favorable outcomes than the non-resilient types. These outcomes included higher subjective well-being and better (mental) health (Bohane et al., 2017; Henning et al., 2017; Isler et al., 2016; Leikas \& Salmela-Aro, 2014; Steca et al., 2010), higher self-esteem (Leikas \& Salmela-Aro, 2014; Pilarska, 2018; Scholte et al., 2005), higher social competencies, social support, and relationship success (Meeus et al., 2011; Scholte et al., 2005; van Aken \& Semon Dubas, 2004; Yu et al., 2014a, 2014b), higher educational and professional attainment (De Fruyt, 2002; Leikas \& Salmela-Aro, 2014), and lower delinquency (Akse et al., 2007a; Klimstra et al., 2010). In particular, psychopathological symptoms such as anxiety and depression were lower in resilients versus non-resilients (Akse et al., 2007b; Klimstra et al., 2010; Leikas \& Salmela-Aro, 2014; Meeus et al., 2011; Spinhoven et al., 2016).

However, both research traditions have surprisingly rarely been combined: How the between-person conceptualization of resilience (i.e., the resilient Big Five personality type) relates to the phenomenon of resilience from a within-person perspective remains unresolved. That is, whether the resilient (vs. non-resilient) personality type predicts less unfavorable and more favorable changes in psychopathological symptoms (e.g., a weaker symptom increase and better recovery) around stressful events requires further investigation.

Resolving this issue is crucial for the construct validation of the resilient personality type: Are people with this type less likely to experience a symptom escalation in response to future stressors, as the type label suggests? In this case, personality type information could improve an early identification of individuals who are at increased risk for mental health problems due to adversities and might profit from targeted interventions. The type approach appears particularly promising in this regard because it combines information on all Big Five simultaneously in a parsimonious way. It does not 
focus on all trait combinations that are theoretically possible but only on those that are empirically relevant in a population (e.g., resilients vs. non-resilients; Asendorpf et al., 2001). From both a theoretical and applied perspective, there is thus a world to win by combining both perspectives.

\section{Aims}

Based on data from a large and nationally representative panel study from the Netherlands, we analyzed nuanced within-person changes in internalizing psychopathological symptoms around four major stressful life events (unemployment, disability, divorce, and widowhood). We not only modeled gradual symptom changes in the years after the respective stressful event (socialization effects), which have often been the focus of past research efforts (Bonanno \& Diminich, 2013; Galatzer-Levy et al., 2018). Like more recent approaches (Infurna \& Luthar, 2016, 2017), we also analyzed gradual symptom changes in the years before the event (anticipation effects). We did so because event-related stress might already increase before the actual event happens (e.g., because people anticipate its occurrence and start to prepare for it; Infurna \& Luthar, 2016, 2017; Kim \& von Dem Knesebeck, 2016; Vable et al., 2015). Moreover, we modeled post-event short-term effects to account for abrupt and transient symptom changes in the first year after the event (vs. all other years) as well as post-event long-term effects to test for enduring long-term symptom changes in the years after the event versus before. Over and above these within-person symptom changes, we considered selection effects, that is, symptom differences between individuals who ever experienced the event and individuals who did not (neither during nor before the study). We chose these different types of event-related predictors consistent with previous research on changes in personality and life satisfaction around major life events (Asselmann \& Specht, 2020a, 2020b, 2021; Denissen et al., 2019). Our modeling approach allowed us to simultaneously analyze selection effects as well as continuous and discontinuous short- and long-term changes in mental health before and after the respective event. Our models were adjusted for sociodemographic factors (i.e., gender and age) and potential methodological confounders (i.e., testing and sampling effects).

Furthermore, we analyzed whether the examined symptom differences and changes (i.e., selection, anticipation, socialization, post-event short-term, and post-event longterm effects) varied by personality type (i.e., resilients vs. non-resilients). Because personality was assessed repeatedly, people's type status may have changed over time. Thus, three sets of analyses were conducted. First, we compared initial resilients to initial non-resilients. That is, we referred to people's initial type status based on their first personality assessment. Second, we compared only stable resilients to stable non-resilients (i.e., individuals who were consistently classified as resilients or as non-resilients at each personality assessment throughout the study). Third, we compared the former group of stable resilients to unstable resilients (i.e., individuals who were initially classified as 
resilients based on their first personality assessment but as non-resilients at a later point of time).

To broaden our understanding of event-related symptom changes beyond internalizing symptoms, we additionally explored changes in substance use around each event. We also modeled changes in perceived job insecurity before unemployment to gain further insights into pre-event distress and anticipatory symptom changes. Moreover, we compared different subtypes of non-resilients (i.e., over- and undercontrollers). Please note that these supplemental and exploratory analyses were not included in our preregistration.

In our preregistration, we intended to also analyze life satisfaction and self-esteem as outcomes. However, to not overstrain the length and density of the paper and avoid overlaps with previous LISS publications (Bleidorn et al., 2021; Denissen et al., 2019), we only focused on internalizing symptoms in this paper.

\section{Hypotheses}

Our hypotheses on within-person symptom trajectories before and after the onset of unemployment, disability, divorce, and widowhood were as follows ${ }^{1}$ : Internalizing symptoms increase gradually in the years before and decrease gradually in the years after the respective event. Internalizing symptoms are particularly high in the first year after the event (vs. all other years) and higher in the years after versus before the event (because people often do not recover completely). In other words, we expected positive anticipation effects, negative socialization effects, positive post-event short-term effects, and positive post-event long-term effects.

Our hypotheses concerning the role of personality types for within-person symptom trajectories around stressful events were as follows: Initial resilients versus initial non-resilients, stable resilients versus stable non-resilients, and stable versus unstable resilients experience less unfavorable and more favorable symptom changes around each event. In other words, we expected the anticipation, post-event short-term, and post-event long-term effects to be weaker and the socialization effects to be stronger in initial resilients versus initial non-resilients, stable resilients versus stable non-resilients, and stable versus unstable resilients.

\footnotetext{
1) In our preregistration (https://osf.io/svye7), we 1) had no hypothesis with respect to symptom differences in the first year after the respective event (no post-event short-term effects), and 2) hypothesized that internalizing symptoms would increase gradually in the years after the event (positive socialization effects). However, because previous research has shown that symptoms tend to be most pronounced around the respective stressful event and decrease thereafter (e.g., Luhmann et al., 2012), it is also possible to hypothesize that internalizing symptoms 1) are most pronounced in the first year after the event (positive post-event short-term effects) and 2) decrease in the years thereafter (negative socialization effects). Please note that these hypotheses deviate from our preregistration.
} 
Selection effects, changes in substance use around each event, changes in job insecurity before unemployment, and differences between subtypes of non-resilients (i.e., overand undercontrollers) were analyzed exploratory (i.e., without any a-priori hypotheses).

\section{Method}

\section{Study Sample}

Our data come from the Longitudinal Internet Studies for the Social Sciences (LISS) panel (Scherpenzeel, 2011; Van der Laan, 2009). The LISS is based on a general population sample of adults, which stems from a nationally representative sample of households drawn from the Dutch population register by Statistics Netherlands. Further details have been previously presented (Scherpenzeel \& Das, 2011).

The LISS panel started in 2007 and is still ongoing. In this paper, we consider information until 2019, the most recent wave so far. The panel was regularly replenished with refreshment cohorts to counteract attrition (Lugtig, 2014). When refreshment cohorts were sampled, information on the associations between sociodemographics (household type, age, ethnicity) and response rates was used to oversample previously underrepresented groups (see https://www.lissdata.nl/lissdata/about-panel/sample-and-recruitment). The consequence for the statistical analyses is that only the initial sample from 2007 (the largest cohort so far) could participate in the entire study from 2007 until 2019. For this cohort, the time resolution and statistical power is thus the largest.

Every year (except for 2014 and 2019), panel members provided information on internalizing symptoms (eleven waves so far). Approximately every two years, panel members provided information on their personality. The exact years in which information on personality was assessed depended on the year in which people had entered the study (planned missingness). Our statistical approach based on multilevel analyses can deal with this planned missingness (i.e., missing information on internalizing symptoms and personality for individual people in individual years).

The LISS panel is conducted in line with the legal requirements in the Netherlands and complies with the Helsinki Declaration of 1975, as revised in 2013. Because our study is based on a secondary analysis of existing data, obtaining ethical approval from an institutional IRB was not necessary.

\section{Transparency, Openness, and Reproducibility}

In previous LISS publications, the authors studied 1) transactions between cultural activities and openness (Schwaba \& Bleidorn, 2019; Schwaba et al., 2018), 2) changes in the Big Five traits and life satisfaction before and after mature adult role transitions (i.e., paid employment, childbirth, marriage, and volunteering) and stressful life events (i.e., unemployment, disability, divorce, and widowhood; Denissen et al., 2019), as well as 3) 
changes in self-esteem before and after divorce (Bleidorn et al., 2021). None of these previous publications overlap with the current study because they 1) did not focus on mental health as outcome, 2) did not include personality types as predictor, and 3) did not examine whether changes in mental health vary by personality type.

\section{Assessment of Major Stressful Life Events}

Throughout the study, panel members completed monthly surveys on their work status (14 options, including "paid employment”, "job seeker following job loss”, "job disability”, and "voluntary work") and marital status (five options, including "married", "divorced", and "widowed"). Based on this status information and consistent with previous research (Denissen et al., 2019), we created dummy variables to code whether and when (i.e., in which year and month) individuals experienced the onset of unemployment, disability, divorce, and widowhood. In participants who experienced the same event more than once during the study, the earliest occurrence was coded.

\section{Assessment of Personality}

Approximately every two years, the Big Five traits extraversion, agreeableness, conscientiousness, emotional stability, and openness were assessed with the International Personality Item Pool (IPIP; Goldberg, 1992). The IPIP consists of 50 items, which are used to describe individuals. Participants are asked to indicate their agreement with these descriptions using a response between 1 (very inaccurate) and 5 (very accurate). Example items are "Talk to a lot of different people at parties" (extraversion), "Sympathize with others' feelings" (agreeableness), "Pay attention to details" (conscientiousness), "Am relaxed most of the time" (emotional stability), and "Have a vivid imagination" (openness). The exact years in which personality was assessed varied across different cohorts, which entered the panel in different years (see Table 1 for more detailed information). Averaged across all waves, internal consistencies ranged from .76 for agreeableness to .88 for extraversion. 


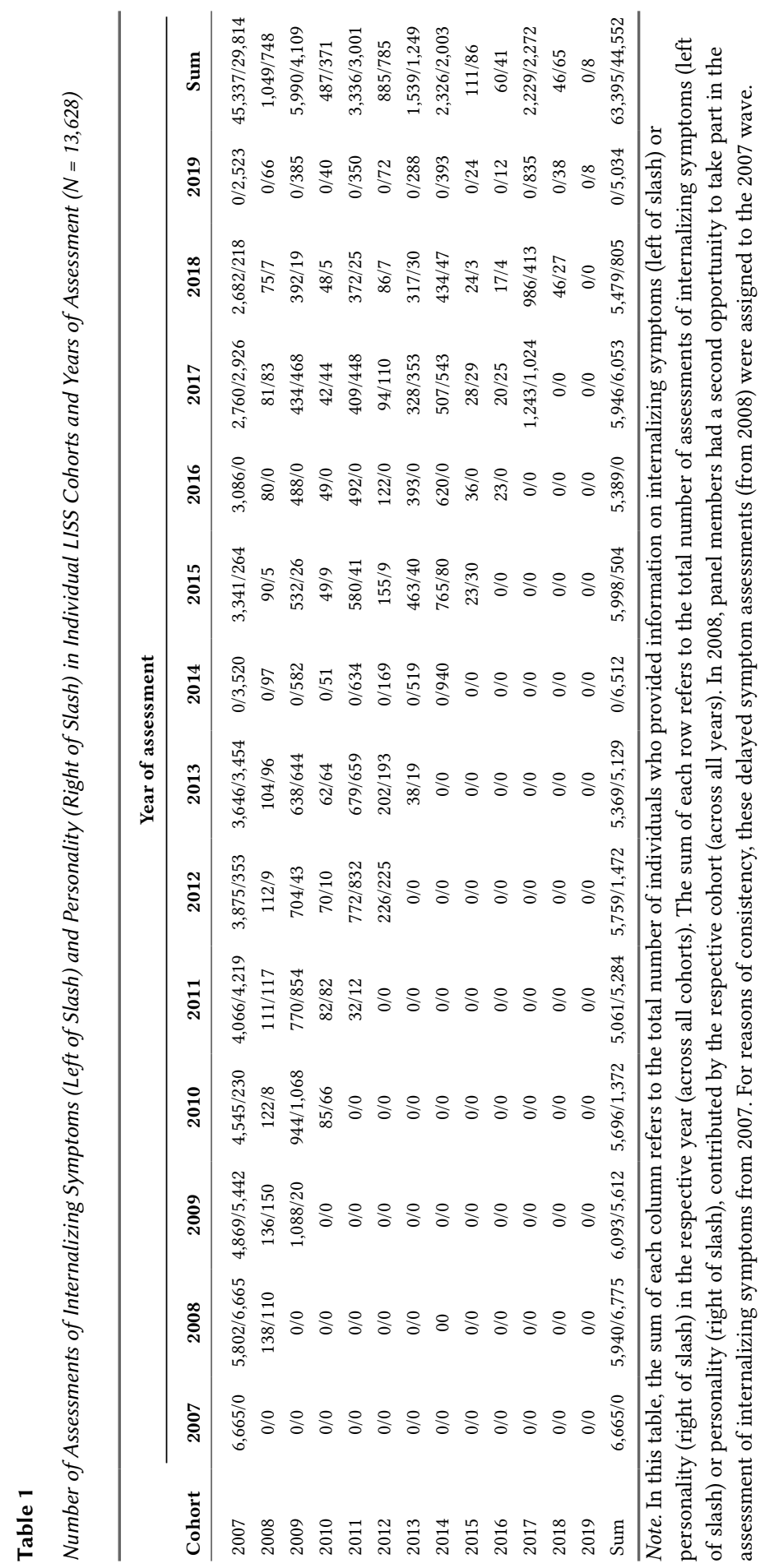




\section{Assessment of Internalizing Symptoms}

Every year (except for 2014 and 2019), information on internalizing symptoms (anxiety and depression) was assessed with the five-item Mental Health Inventory (MHI-5; Ware \& Sherbourne, 1992). In the MHI-5, participants are asked how often they felt anxious, so down that nothing could cheer them up, calm and peaceful, depressed, and gloomy, as well as happy during the past month. Items are labeled from 1 (never) to 6 (continuously). Averaged across all waves, the internal consistency was .85 .

\section{Statistical Analysis}

Personality types were obtained with latent profile analyses in Latent GOLD 5.0 (Vermunt \& Magidson, 2013). We first transformed the data from wide to long format and then classified participants based on their Big Five trait scores consistent with previous research (Alessandri et al., 2014; Asendorpf et al., 2001). Individuals who provided information on their personality more than once during the study were classified multiple times, thus being able to examine the role of type stability. Our aim was not to identify the class solution that optimally represented the multivariate distribution of the Big Five trait scores but to identify the most parsimonious model (with the fewest classes) that contained a resilient type. We investigated whether adding a class led to a better model fit, as indicated by a lower Bayesian information criterion (BIC; Schwarz, 1978) and a significant bootstrap likelihood ratio test (BLRT; Nylund et al., 2007).

Findings revealed that a two-class solution outperformed a one-class solution (in which no classes were distinguished), as indicated by a much lower BIC $\left(\mathrm{BIC}_{1 \text { class }}=\right.$ $379,201.57, \mathrm{BIC}_{2}$ classes $\left.=363,848.21, \Delta \mathrm{BIC}=15,353.36\right)$ and a significant BLRT $(p<.001)$. This two-class solution contained a clearly identifiable resilient type with above-average scores and a non-resilient type with below-average scores across the Big Five. Therefore, we chose this model for the analyses. In resilients/non-resilients, the means were 3.68/2.94 for extraversion, 4.16/3.66 for agreeableness, 3.98/3.51 for conscientiousness, $3.77 / 3.20$ for emotional stability, and 3.78/3.27 for openness, respectively.

Selection effects and within-person symptom trajectories around the respective stressful event were modeled using multilevel analyses with measurement occasions (Level 1) nested within persons (Level 2) and households (Level 3) using the LME4 package in $\mathrm{R}$ (Bates et al., 2014). Our analyses follow the approach of a recent study (Denissen et al., 2019). Specifically, we simultaneously regressed the standardized score of internalizing symptoms on five event-related predictors (selection, anticipation, post-event short-term, post-event long-term, and socialization). These predictors coded whether individuals had ever experienced the event or not and how the event (if experienced) was temporarily related to the respective symptom assessment (in monthly increments). This approach allowed us analyzing symptom differences between individuals who did and did not experience the event (selection effects) and within-person symptom changes 
around the event (if experienced). Each model was adjusted for gender (to account for differences between women and men), linear age (to account for linear age effects), quadratic age (to account for non-linear age effects), testing effects (to account for effects due to repeated symptom assessments), and sampling effects (to account for differences between individuals who experienced the respective event during vs. before the study period). Linear and quadratic age were included because personality and mental health are both associated with age and these associations are rarely linear (Denissen et al., 2019; Specht et al., 2011; Westerhof \& Keyes, 2010). Table S1 (see Supplementary Materials) specifies how each predictor was defined and coded.

In addition, we tested whether the selection effects and event-related symptom changes varied by personality type. That is, we simultaneously added five interaction terms between the respective event-related predictor (selection, anticipation, post-event short-term, post-event long-term, and socialization) and a personality type dummy variable (Level 2 predictor) to our model. Three sets of analyses were conducted. First, we compared initial resilients (coded with 1) to initial non-resilients (coded with 0) based on their first personality assessment. Second, we compared only stable resilients (coded with 1 ) to stable non-resilients (coded with 0 ). In these analyses, only individuals who participated in at least two personality assessments and were consistently classified as resilients or as non-resilients at each assessment were considered. Third, we compared the former group of stable resilients (coded with 1) to unstable resilients (i.e., individuals who were initially classified as resilients but as non-resilients at a later point of time; coded with 0 ).

We built separate models per event (unemployment, disability, divorce, and widowhood) and modeled the effects as fixed effects. We corrected our $p$-values for false discovery rate using the "BY" method (Benjamini \& Hochberg, 1995). After correction for multiple testing, we used an alpha level of .10 for confirmatory analyses and an alpha level of .05 for exploratory analyses, which approximately corresponds to an uncorrected $p<.001$ in the former and an uncorrected $p<.01$ in the latter case. Analogously, we computed $99.9 \%$ confidence intervals for confirmatory analyses and $99.0 \%$ confidence intervals for exploratory analyses (i.e., on changes in substance use around each event, changes in job insecurity before unemployment, and differences between subtypes of non-resilients). Confidence intervals were estimated by computing the $z$-value that belongs to the focal $p$-value (i.e., $p=.001$ for confirmatory analyses and $p=.010$ for exploratory analyses) and by multiplying the corresponding $z$-value with the standard error of the multilevel estimates.

\section{Power Analysis}

The power analysis was conducted with the SIMR package in R (Green \& MacLeod, 2016). Because our data structure was complex and our multilevel models included multiple predictors, it was impossible to estimate power in a straightforward way. Consistent 
with previous LISS publications (Denissen et al., 2019), we thus based our power analysis on the parameter with the fewest underlying data points, that is, the difference between resilients and non-resilients in the first year of widowhood.

Specifically, we selected all individuals who experienced the onset of widowhood and then computed the smallest detectable effect size when setting the power at $80 \%$. Our simulation results revealed that a regression coefficient of .60 (which is roughly equivalent to Cohen's d because of the dummy coding) for the difference of the post-event short-term effect between resilients and non-resilients (concerning widowhood) would be detected with $79 \%$ power. Importantly, this estimate is based on the "weakest link", which means that all other effects would be detected with substantially more power. For example, a regression coefficient of .40 for the difference of the post-event short-term effect between resilients and non-resilients would be detected with $99 \%$ power. Roughly speaking, these results suggest that our study is sufficiently powered to detect moderate and also large effects for estimates based on comparatively few data points as well as even very small effects for estimates based on a higher number of data points.

\section{Results}

\section{Sample Characteristics}

Table 1 shows in which years individual cohorts entered the LISS panel and provided information on internalizing symptoms and personality, respectively. Of the total sample $(N=22,115), 13,628$ individuals provided information on internalizing symptoms and 12,551 individuals additionally provided information on their personality at least once during the study. Based on their first personality assessment, 5,374 (42.82\%) were classified as initial resilients and 7,177 (57.18\%) as initial non-resilients. Test-retest correlations of personality types across waves indicated substantial stability (Table S2, see Supplementary Materials).

Of those with at least two personality assessments $(N=9,883,78.74 \%), 2,605(26.36 \%)$ were consistently classified as resilients and 3,968 (40.15\%) were consistently classified as non-resilients throughout the entire study (stable resilients and non-resilients, respectively). Furthermore, 1,649 (16.69\%) were classified as resilients based on their first personality assessment but as non-resilient at a later point of time (unstable resilients). Sample characteristics of these groups are presented in Table 2. 
Table 2

Sample Characteristics

\begin{tabular}{lccccc}
\hline Sample characteristic & $\begin{array}{c}\text { Initial } \\
\text { resilients }\end{array}$ & $\begin{array}{c}\text { Initial non- } \\
\text { resilients }\end{array}$ & $\begin{array}{c}\text { Stable } \\
\text { resilients }\end{array}$ & $\begin{array}{c}\text { Stable non- } \\
\text { resilients }\end{array}$ & $\begin{array}{c}\text { Unstable } \\
\text { resilients }\end{array}$ \\
\hline$N$ & 5,374 & 7,177 & 2,605 & 3,968 & 1,649 \\
Internalizing symptoms (M) & 2.14 & 2.49 & 2.03 & 2.55 & 2.26 \\
Age (M) & 47.87 & 45.08 & 49.25 & 46.37 & 48.96 \\
Female gender (\%) & 58.08 & 52.11 & 58.62 & 51.56 & 56.46 \\
Unemployment $^{\mathrm{a}}(\%)$ & $9.34 / 8.11$ & $9.71 / 8.15$ & $9.56 / 8.64$ & $9.85 / 8.47$ & $11.4 / 10.37$ \\
Disability $^{\mathrm{a}}(\%)$ & $4.95 / 2.61$ & $6.51 / 2.95$ & $4.64 / 2.53$ & $7.28 / 3.33$ & $6.85 / 3.7$ \\
Divorce $^{\mathrm{a}}(\%)$ & $11.89 / 2.31$ & $9.63 / 2.1$ & $12.36 / 2.19$ & $9.95 / 2.32$ & $12.31 / 3.27$ \\
Widowhood $^{\mathrm{a}}(\%)$ & $6.29 / 1.69$ & $5.91 / 1.52$ & $6.79 / 1.46$ & $6.68 / 1.76$ & $7.1 / 3.09$ \\
\hline
\end{tabular}

${ }^{\text {aLeft }}$ of slash: percentage of individuals who ever experienced the event, right of slash: percentage of individuals who experienced the event during the study.

\section{Selection Effects and Event-Related Changes in Internalizing Symptoms}

Selection effects and event-related changes in internalizing symptoms are shown in Table 3. In terms of selection effects, we found that internalizing symptoms were higher in individuals who ever experienced the onset of unemployment $(b=0.29)$, disability $(b=$ $0.70)$, or divorce $(b=0.20)$ compared to individuals who did not. In terms of event-related symptom changes, we found that internalizing symptoms increased gradually in the years before the onset of unemployment $(b=0.13)$, disability $(b=0.39)$, divorce $(b=0.45)$, and widowhood $(b=0.20)$ (anticipation effects). Internalizing symptoms were higher in the first year after the onset of disability $(b=0.27)$ and widowhood $(b=0.56)$ compared to all other years (post-event short-term effects). Moreover, internalizing symptoms decreased suddenly after the onset of unemployment $(b=-0.12)$ and disability $(b=-0.15)$ (post-event long-term effects) and decreased gradually in the years after getting divorced (socialization effect: $b=-0.25$ ). 


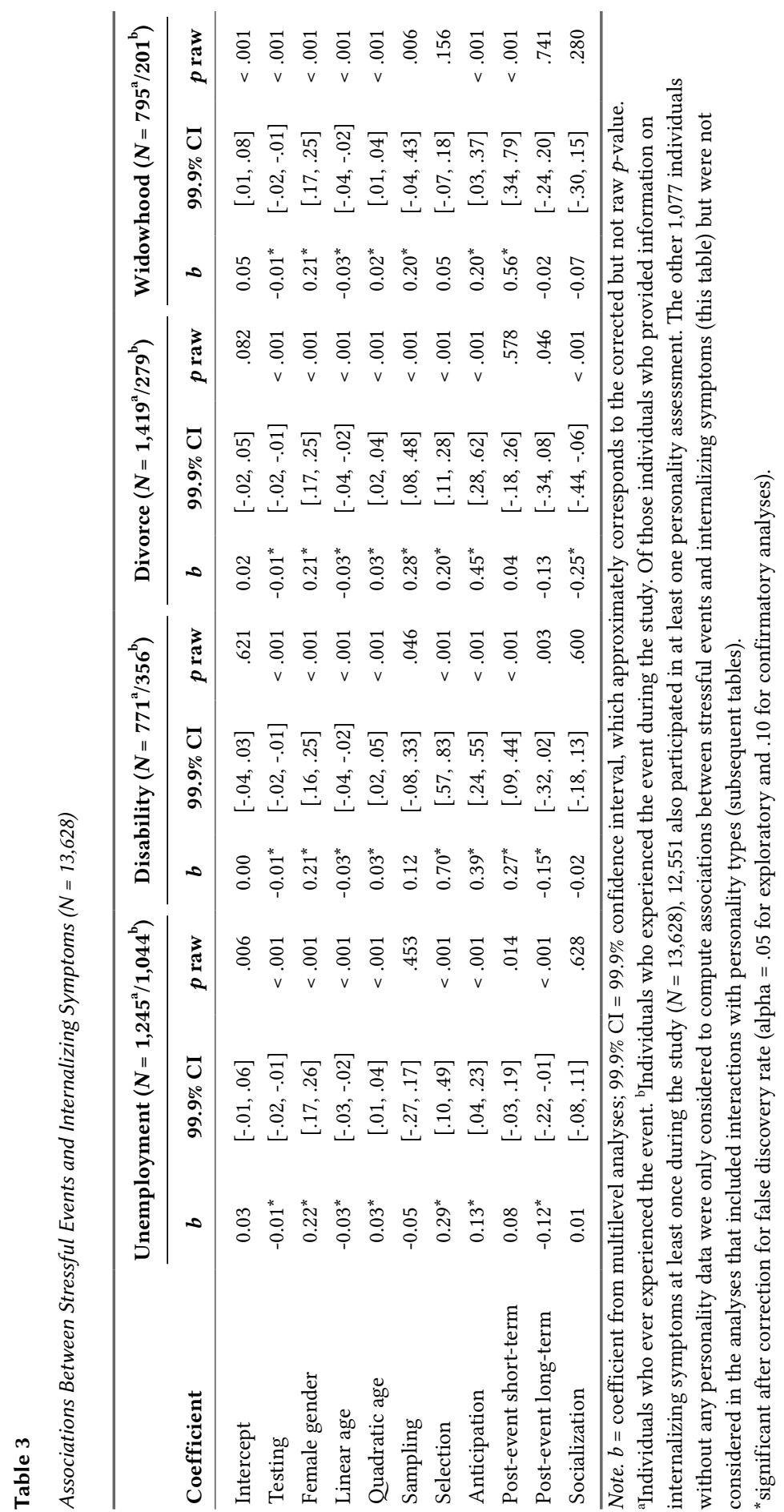


Average smoothed trajectories of internalizing symptoms in the years around the respective stressful event are visualized in Figure 1. Confirming our statistical analyses, internalizing symptoms increased as individuals approached the event, but the timing and amplitude of this increase varied. The symptom increase before the onset of widowhood started comparatively late, and the symptom increase around the onset of unemployment was only half as large compared to the other events. Importantly, internalizing symptoms increased until each event but decreased thereafter, indicating that people, on average, recovered from event-related symptoms in the aftermath of the respective stressful experience.

\section{Figure 1}

Average Smoothed Trajectories of Internalizing Symptoms From Five Years Before Until Five Years After the Onset of Unemployment (Red Line), Disability (Green Line), Divorce (Blue Line), and Widowhood (Purple Line) in Individuals Who Experienced the Respective Event During the Study

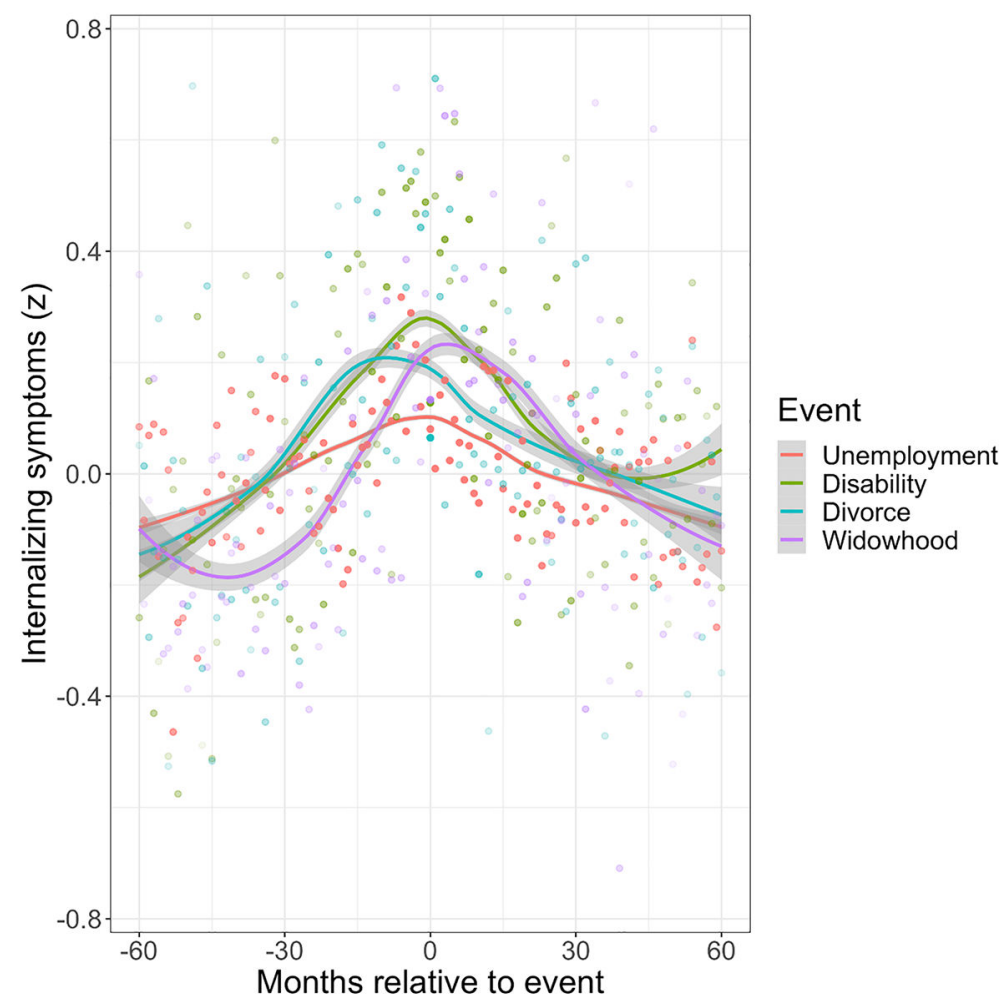

Note. Symptom scores were standardized before inclusion in the graph. The dots indicate average symptom levels in specific months around the event (in individuals who provided information on internalizing symptoms in this month). The color of the dots indicates the number of underlying data points with darker dots representing more cases compared to lighter dots. 


\section{The Role of Personality Types}

\section{Initial Resilients Versus Initial Non-Resilients}

Examining the role of people's initial personality types (based on their first personality assessment, Table 4) revealed that internalizing symptoms were lower in initial resilients compared to initial non-resilients (personality type main effects: $b=-0.38$ to -0.40$)^{2}$. In terms of selection effects, we found that the symptom difference between individuals who did versus did not get divorced (with higher symptoms in people who got divorced) was smaller in initial resilients versus initial non-resilients (personality type $\mathrm{x}$ selection effect: $b=-0.16)^{2}$. However, most interactive effects did not reach statistical significance. That is, other selection effects and the event-related symptom trajectories did not vary significantly by initial personality type.

\section{Stable Resilients Versus Stable Non-Resilients}

Like our findings on initial personality types, examining the role of stable personality types (Table S4, see Supplementary Materials) revealed that internalizing symptoms were lower in stable resilients compared to stable non-resilients (personality type main effects: $b=-0.57$ to -0.62$)^{2}$.

In terms of selection effects, we found that the symptom difference between individuals who did versus did not experience the onset of disability (with higher symptoms in people who became disabled) was smaller in stable resilients versus stable non-resilients (personality type $\mathrm{x}$ selection effect: $b=-0.29)^{2}$. Similarly, the symptom difference between individuals who did versus did not get divorced (with higher symptoms in people who got divorced) was smaller in stable resilients versus stable non-resilients (personality type x selection effect: $b=-0.19)^{3}$. However, no other interactive effects were found. That is, the selection effects for unemployment and widowhood did not vary significantly by stable personality type.

In terms of event-related symptom trajectories, our findings revealed that the gradual symptom increase before the onset of unemployment was lower in stable resilients versus stable non-resilients (personality type $\mathrm{x}$ anticipation effect: $b=-0.23)^{3}$, see Figure 2. Furthermore, the symptom rebound after the onset of disability was stronger in stable resilients versus stable non-resilients (personality type x post-event long-term effect: $b$ $=-0.39)^{3}$, see Figure 3. However, no other interactive effects were found. In other words, other event-related symptom changes did not vary significantly by stable personality type.

\footnotetext{
2) These effects remained significant after adjustment for social desirability (see Table S3 and Table S5 in Supplementary Materials).

3) These effects were marginally significant after adjustment for social desirability (see Table S5 in Supplementary Materials).
} 


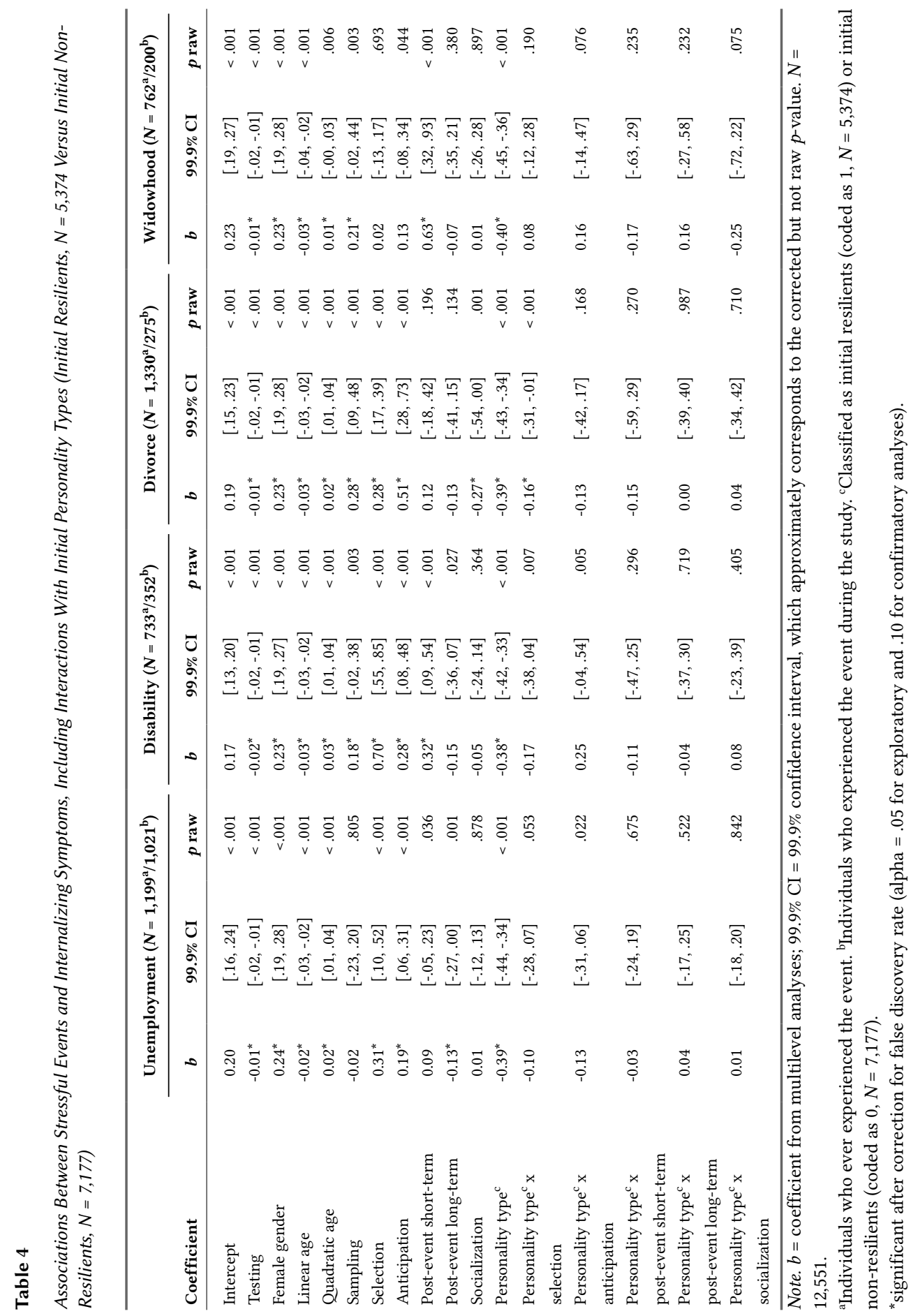




\section{Figure 2}

Average Smoothed Trajectories of Internalizing Symptoms From Five Years Before Until Five Years After the Onset of Unemployment in Individuals Who Experienced This Event During the Study and Were Classified as Stable Resilients (Blue Line) or Non-Resilients (Red Line)

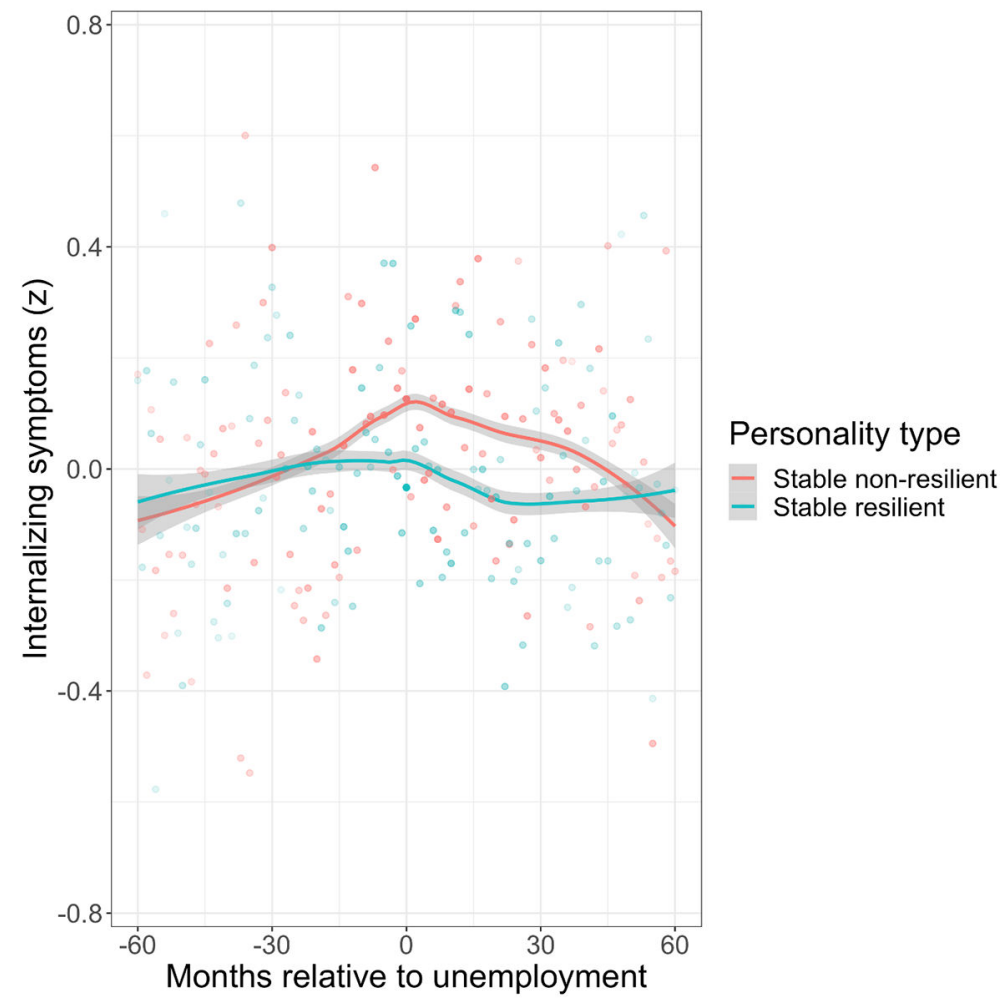

Note. Symptom scores were standardized before inclusion in the graph. The dots indicate average symptom levels in specific months around the onset of unemployment. The color of the dots indicates the number of underlying data points with darker dots representing more cases compared to lighter dots. 


\section{Figure 3}

Average Smoothed Trajectories of Internalizing Symptoms From Five Years Before Until Five Years After the Onset of Disability in Individuals Who Experienced This Event During the Study and Were Classified as Stable Resilients (Blue Line) or Non-Resilients (Red Line)

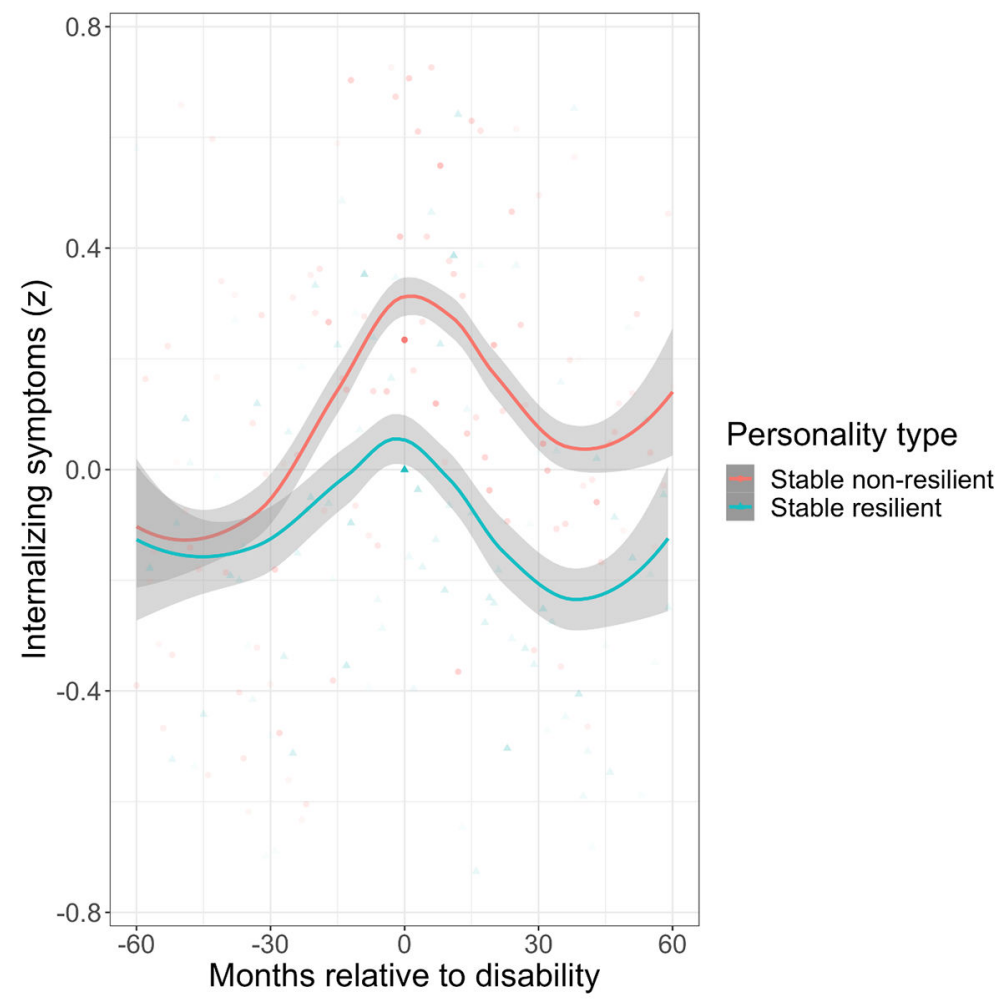

Note. Symptom scores were standardized before inclusion in the graph. The dots indicate average symptom levels in specific months around the onset of disability. The color of the dots indicates the number of underlying data points with darker dots representing more cases compared to lighter dots.

\section{Stable Resilients Versus Unstable Resilients}

Comparing stable resilients to unstable resilients (Table S6, see Supplementary Materials) revealed that internalizing symptoms were lower in stable resilients compared to unstable resilients (personality type main effects: $b=-0.26$ to -0.28 ). Furthermore, stable resilients experienced a stronger symptom rebound after the onset of disability than unstable resilients (personality type x post-event long-term effect: $b=-0.58$ ). However, no other interactive effects were found. That is, the selection effects and other event-related symptom changes did not differ significantly between stable and unstable resilients. 


\section{Overcontrollers Versus Undercontrollers}

In exploratory analyses, we further compared stable overcontrollers to stable undercontrollers (Table S7, see Supplementary Materials). Consistent with previous evidence that overcontrollers, in particular, tend to be susceptible to internalizing symptoms (Klimstra et al., 2010; Leikas \& Salmela-Aro, 2014; Meeus et al., 2011; van Aken \& Semon Dubas, 2004), we found that internalizing symptoms were higher in overcontrollers compared to undercontrollers (personality type main effects: $b=0.45$ to 0.51 ). However, the selection effects and event-related symptom changes did not differ between overcontrollers and undercontrollers.

\section{The Role of Job Insecurity}

To better understand why internalizing symptoms already increased in large parts before the actual stressful event occurred (Kim \& von Dem Knesebeck, 2016), we conducted supplemental analyses to analyze changes in perceived job insecurity before the onset of unemployment. As shown in Table S8 and Figure S1 (see Supplementary Materials), job insecurity was lower in stable resilients compared to stable non-resilients (personality type main effect: $b=-0.29)^{4}$, and increased before people lost their job (anticipation effect: $b=0.56$ ). Importantly, this anticipatory increase of job insecurity did not differ significantly between stable resilients and stable non-resilients (no significant personality type $\mathrm{x}$ anticipation effect).

Taken together, these findings suggest 1) that the onset of unemployment indicated the culmination of stress due to increasing job insecurity and 2) that resilients and nonresilients experienced this stress in a similar way. However, compared to non-resilients, resilients seemed to be able to cope with this distress more successfully. This might explain our finding that stable resilients experienced a lower increase of internalizing symptoms than stable non-resilients before losing their job (see Figure 2).

\section{Associations Between Stressful Events and Substance Use}

We not only focused on internalizing symptoms but also explored how illicit drug and problematic alcohol use changed around stressful events. These analyses were based on dichotomous outcomes, so that regression coefficients indicate log-odds.

Substance use neither differed between initial resilients and initial non-resilients nor between stable resilients and stable non-resilients (no significant personality type main effects), so we proceeded without modeling the role of personality types ${ }^{5}$. As presented

\footnotetext{
4) Our supplemental analyses refer to stable (instead of initial) personality types because mental health differences were most pronounced in stable resilients versus stable non-resilients.

5) Examining whether event-related changes in substance use varied by personality type was also impossible due to convergence problems.
} 
in Table S9 (see Supplementary Materials), illicit drug use was higher in individuals who ever experienced the onset of unemployment $(b=0.84)$ or disability $(b=1.17)$ versus individuals who did not (selection effects). Illicit drug use increased gradually in the years before (anticipation effect: $b=0.96$ ) and decreased gradually in the years after (socialization effect: $b=-0.96$ ) people lost their job. In people who experienced the onset of widowhood, illicit drug use increased gradually in the following years (socialization effect: $b=2.21$ ).

As shown in Table S10 (see Supplementary Materials), problematic alcohol use was higher in people who ever got versus did not get divorced (selection effect: $b=0.77$ ). However, there was no evidence for event-related changes in problematic alcohol use.

\section{Discussion}

Based on longitudinal data from a large and nationally representative sample from the Netherlands, this study examined whether nuanced within-person changes in internalizing symptoms around four major stressful life events varied by personality type (i.e., resilients vs. non-resilients). In terms of event-related symptom changes, our main finding was that internalizing symptoms increased before and decreased (i.e., recovered) after the respective stressful event. Regarding personality types, we found that stable resilients experienced a weaker symptom increase before and recovered better from certain stressful events than stable non-resilients. For the first time, we could thus demonstrate that the between-person conceptualization of a resilient personality type corresponds to the phenomenon of resilience from a within-person perspective, at least for certain events and stable types. However, most within-person symptom changes around stressful events did not vary by personality type, especially when considering initial (instead of stable) personality types, based on people's first personality assessment.

In terms of event-related symptom trajectories, our plots and statistical results were largely consistent with our hypotheses and converged on the conclusion that internalizing symptoms increased in the years before and decreased in the years after the onset of unemployment, disability, divorce, and widowhood. Similarly, our exploratory analyses on substance use revealed that illicit drug use increased before and decreased after the onset of unemployment. Problematic alcohol use, however, did not change. That is, on average, people seem to recover from transient anxiety and depressive symptoms as well as problematic substance use due to such stressful experiences. That said, this average pattern of recovery was relatively slow, partially incomplete, and not found consistently. For example, after the onset of widowhood, illicit drug use increased considerably. These findings are in line with previous evidence for changes in subjective well-being (Denissen et al., 2019; Infurna \& Luthar, 2016, 2017; Lucas, 2005; Lucas et al., 2004; Luhmann et al., 2012), self-esteem (Bleidorn et al., 2021; Luciano \& Orth, 2017) and psychopathological symptoms (Kim \& von Dem Knesebeck, 2016; Recksiedler \& Stawski, 
2019; Siflinger, 2017; Vable et al., 2015) around the onset of unemployment, disability, divorce, or widowhood. We considerably extended this previous research by analyzing event-related symptom changes in a much finer resolution (i.e., in monthly increments).

Our most important implication is that within-person symptom trajectories around stressful events varied - at least partially - by personality type. Consistent with our hypotheses, stable resilients experienced a weaker increase of internalizing symptom before unemployment than stable non-resilients. They also experienced a stronger symptom rebound after the onset of disability, indicating better recovery. Moreover, internalizing symptoms were higher in individuals who did versus did not experience stressful events, but this symptom difference was lower in resilients versus non-resilients. Based on previous research (Herzberg \& Roth, 2006; Leikas \& Salmela-Aro, 2014; Meeus et al., 2011), one might speculate whether resilients tend to possess a wider range of cognitive, emotional, and socio-economic abilities and resources than non-resilients. Such resources might enable them to cope with adverse experiences and related stress (e.g., accumulating job insecurity before unemployment) more successfully, which could explain our results. However, several hypotheses were not confirmed. That is, only specific symptom trajectories around the onset of unemployment and disability (but not divorce and widowhood) differed between resilients and non-resilients, and these differences were only found for stable but not initial personality types and remained only marginally significant after adjustment for social desirability. These results underline the importance of type stability for the prediction of future adjustment based on personality types. Moreover, these findings emphasize that resilience depends on the kind of adversity that people are faced with. Future research is needed to zoom into the underlying processes that might explain why certain symptom changes around stressful events vary by personality type and others do not. This research may also investigate whether specific thoughts, feelings, and behaviors or traits (e.g., emotional stability) drive the beneficial effects of the resilient personality type on mental health under stressful circumstances. Relatedly, future research (e.g., experience sampling studies) may investigate whether other operationalizations of resilience (e.g., ego-resilience) relate to higher flexibility to adjust to specific situations and thus predict higher within-person resilience.

\section{Strengths and Limitations}

Our study has several strengths: We used longitudinal data from a large, socio-demographically diverse, and nationally representative household panel study from the Netherlands $(N=13,628)$, which covered the entire adult lifespan. Personality and different indicators of mental health were measured with established and highly reliable measures. Information on life events was obtained monthly and mental health was assessed yearly from 2007 to 2019. Due to the large sample, hundreds of individuals experienced the onset of unemployment, disability, divorce, and widowhood during the study. This ena- 
bled us to study complex symptom changes before and after these events, including interactions with personality types.

However, our study is not without limitations. First, the LISS panel was regularly replenished with refreshment cohorts, which entered the panel in different years. Therefore, only the initial sample from 2007 could participate in the entire study (twelve years so far). Second, our study primarily focused on internalizing symptoms (i.e., anxiety and depression). In supplemental analyses, we also considered externalizing symptoms (i.e., illicit drug and problematic alcohol use), but due to model convergence issues, we were not able to test whether nuanced changes in substance use varied by personality type. Besides, diagnostic information was assessed via self-report and might have been biased. Third, although unemployment, disability, divorce, and widowhood tend to be stressful experiences for many people, subjective event perceptions might vary substantially between individuals (Luhmann et al., 2020). For example, getting divorced might be highly stressful for some but less stressful for other people depending on their family and financial situation as well as other factors. Fourth, latent class analyses have sometimes been criticized for producing sub-groups that are hard to replicate (Bauer \& Curran, 2003). However, the broad distinction of resilients and non-resilients has been evidenced successfully across different populations and studies and is considered as relatively robust (Alessandri et al., 2014; Asendorpf et al., 2001). Finally, the generalizability of our findings to other regions (outside the Netherlands) and time points might be limited. For example, in countries with poorer safety nets and greater socioeconomic inequality, personality types might differently affect whether people maintain or regain good mental health despite substantial adversity.

\section{Conclusions}

To our knowledge, this study is the first to empirically link two different conceptualizations of within- and between-person resilience. Our results boost the construct validity of the resilient personality type and are highly relevant for developmental, health, and clinical psychology. Especially information on personality types might improve an early identification of high-risk individuals (i.e., non-resilients), who are more susceptible to mental health problems due to work-related adversities and might thus profit from targeted preventions and early interventions. 
Funding: Preparations of this manuscript were supported by the Netherlands Organization for Scientific Research via a Vidi grant (452-14-013) to Theo A. Klimstra.

Acknowledgments: We would like to thank CentERdata (Tilburg University, The Netherlands) for the ability to use the data from the LISS (Longitudinal Internet Studies for the Social sciences) panel.

Competing Interests: The authors have declared that no competing interests exist.

Author Contributions: Eva Asselmann-Idea, conceptualization | Design planning | Research implementation (software, hardware, etc.) | Validation, reproduction, checking | Writing | Supervision, mentoring | Project coordination, administration. Theo A. Klimstra-Idea, conceptualization | Design planning | Research implementation (software, hardware, etc.) | Data analysis | Validation, reproduction, checking | Writing | Feedback, revisions | Supervision, mentoring | Project coordination, administration. Faap f. A. Denissen-Idea, conceptualization | Design planning | Research implementation (software, hardware, etc.) | Visualization (data presentation, figures, etc.) | Data analysis | Validation, reproduction, checking | Writing | Feedback, revisions | Supervision, mentoring | Project coordination, administration.

Ethics Statement: The LISS panel is conducted in line with the legal requirements in the Netherlands and complies with the Helsinki Declaration of 1975, as revised in 2013. Because our study is based on a secondary analysis of existing data, obtaining ethical approval from an institutional IRB was not necessary.

Related Versions: No other previously published versions of this manuscript exist in part or in whole.

Data Availability: A detailed description of all procedures and measures collected in the LISS as well as a list of previous LISS publications can be found at https://www.lissdata.nl. The LISS data are available for researchers from CentERdata after signing a data statement at https://statements.centerdata.nl.

\section{Supplementary Materials}

For this article the following Supplementary Materials are available (for access see Index of Supplementary Materials below).

Via the Open Science Framework (OSF) repository:

- The formal preregistration of the study, including information on the background, aims, hypotheses, study type and design, as well as a detailed analysis plan.

Via the PsychArchives repository:

- Analysis scripts.

- Supplemental findings: Figure S1, Tables S1-S10. 


\section{Index of Supplementary Materials}

Asselmann, E., Klimstra, T. A., \& Denissen, J. J. A. (2019). Supplementary materials to: Unraveling resilience: Personality predicts exposure and reaction to stressful life events [Preregistration documentation]. OSF. https://osf.io/svye7

Asselmann, E., Klimstra, T. A., \& Denissen, J. J. A. (2021). Supplementary materials to: Unraveling resilience: Personality predicts exposure and reaction to stressful life events [Analysis scripts, Figure, and Tables]. PsychOpen GOLD. https://doi.org/10.23668/psycharchives.5184

\section{References}

Akse, J., Hale, B., Engels, R., Raaijmakers, Q., \& Meeus, W. (2007a). Co-occurrence of depression and delinquency in personality types. European fournal of Personality, 21(2), 235-256. https://doi.org/10.1002/per.604

Akse, J., Hale, W. W., III, Engels, R. C., Raaijmakers, Q. A., \& Meeus, W. H. (2007b). Stability and change in personality type membership and anxiety in adolescence. fournal of Adolescence, 30(5), 813-834. https://doi.org/10.1016/j.adolescence.2006.09.007

Alessandri, G., Vecchione, M., Donnellan, B. M., Eisenberg, N., Caprara, G. V., \& Cieciuch, J. (2014). On the cross-cultural replicability of the resilient, undercontrolled, and overcontrolled personality types. Fournal of Personality, 82(4), 340-353. https://doi.org/10.1111/jopy.12065

Asendorpf, J. B., Borkenau, P., Ostendorf, F., \& Van Aken, M. A. (2001). Carving personality description at its joints: Confirmation of three replicable personality prototypes for both children and adults. European fournal of Personality, 15(3), 169-198.

https://doi.org/10.1002/per.408

Asendorpf, J. B., \& Denissen, J. J. (2006). Predictive validity of personality types versus personality dimensions from early childhood to adulthood: Implications for the distinction between core and surface traits. Merrill-Palmer Quarterly, 52(3), 486-513.

https://doi.org/10.1353/mpq.2006.0022

Asendorpf, J. B., \& van Aken, M. A. (1999). Resilient, overcontrolled, and undercontroleed personality prototypes in childhood: Replicability, predictive power, and the trait-type issue. Journal of Personality and Social Psychology, 77(4), 815-832. https://doi.org/10.1037/0022-3514.77.4.815

Asselmann, E., \& Specht, J. (2020a). Taking the ups and downs at the rollercoaster of love: Associations between major life events in the domain of romantic relationships and the Big Five personality traits. Developmental Psychology, 56(9), 1803-1816. https://doi.org/10.1037/dev0001047

Asselmann, E., \& Specht, J. (2020b). Till death do us part: Transactions between losing one's spouse and the Big Five personality traits. Journal of Personality, 88(4), 659-675.

https://doi.org/10.1111/jopy.12517 
Asselmann, E., \& Specht, J. (2021). Testing the Social Investment Principle around childbirth: Little evidence for personality maturation before and after becoming a parent. European fournal of Personality, 35(1), 85-102. https://doi.org/10.1002/per.2269

Asselmann, E., Wittchen, H.-U., Lieb, R., Höfler, M., \& Beesdo-Baum, K. (2016). Does low coping efficacy mediate the association between negative life events and incident psychopathology? A prospective-longitudinal community study among adolescents and young adults. Epidemiology and Psychiatric Sciences, 25(2), 171-180. https://doi.org/10.1017/S204579601500013X

Bates, D., Mächler, M., Bolker, B., \& Walker, S. (2014). Fitting linear mixed-effects models using lme4. arXiv. https://arxiv.org/abs/1406.5823

Bauer, D. J., \& Curran, P. J. (2003). Distributional assumptions of growth mixture models: implications for overextraction of latent trajectory classes. Psychological Methods, 8(3), 338-363. https://doi.org/10.1037/1082-989X.8.3.338

Benjamini, Y., \& Hochberg, Y. (1995). Controlling the false discovery rate: a practical and powerful approach to multiple testing. Fournal of the Royal Statistical Society. Series B. Methodological, 57(1), 289-300. https://doi.org/10.1111/j.2517-6161.1995.tb02031.x

Bleidorn, W., Schwaba, T., Denissen, J. J., \& Hopwood, C. J. (2021). Charting self-esteem during marital dissolution. Fournal of Personality, 89(1), 9-22. https://doi.org/10.1111/jopy.12525

Block, J. (1971). Lives through time. Bancroft Books.

Bohane, L., Maguire, N., \& Richardson, T. (2017). Resilients, overcontrollers and undercontrollers: a systematic review of the utility of a personality typology method in understanding adult mental health problems. Clinical Psychology Review, 57, 75-92.

https://doi.org/10.1016/j.cpr.2017.07.005

Bonanno, G. A., \& Diminich, E. D. (2013). Annual research review: Positive adjustment to adversity-trajectories of minimal-impact resilience and emergent resilience. Fournal of Child Psychology and Psychiatry, and Allied Disciplines, 54(4), 378-401.

https://doi.org/10.1111/jcpp.12021

Caspi, A. (2000). The child is father of the man: personality continuities from childhood to adulthood. fournal of Personality and Social Psychology, 78(1), 158-172. https://doi.org/10.1037/0022-3514.78.1.158

Caspi, A., Harrington, H., Milne, B., Amell, J. W., Theodore, R. F., \& Moffitt, T. E. (2003). Children's behavioral styles at age 3 are linked to their adult personality traits at age 26. Fournal of Personality, 71(4), 495-514. https://doi.org/10.1111/1467-6494.7104001

Caspi, A., Moffitt, T. E., Newman, D. L., \& Silva, P. A. (1996). Behavioral observations at age 3 years predict adult psychiatric disorders: Longitudinal evidence from a birth cohort. Archives of General Psychiatry, 53(11), 1033-1039. https://doi.org/10.1001/archpsyc.1996.01830110071009

Chapman, B. P., \& Goldberg, L. R. (2011). Replicability and 40-year predictive power of childhood ARC types. Fournal of Personality and Social Psychology, 101(3), 593-606. https://doi.org/10.1037/a0024289

De Fruyt, F. (2002). A person-centered approach to P-E fit questions using a multiple-trait model. fournal of Vocational Behavior, 60(1), 73-90. https://doi.org/10.1006/jvbe.2001.1816 
Denissen, J. J., Asendorpf, J. B., \& Van Aken, M. A. (2008). Childhood personality predicts longterm trajectories of shyness and aggressiveness in the context of demographic transitions in emerging adulthood. Fournal of Personality, 76(1), 67-100.

https://doi.org/10.1111/j.1467-6494.2007.00480.x

Denissen, J. J., Luhmann, M., Chung, J. M., \& Bleidorn, W. (2019). Transactions between life events and personality traits across the adult lifespan. fournal of Personality and Social Psychology, 116(4), 612-633. https://doi.org/10.1037/pspp0000196

Galatzer-Levy, I. R., Huang, S. H., \& Bonanno, G. A. (2018). Trajectories of resilience and dysfunction following potential trauma: A review and statistical evaluation. Clinical Psychology Review, 63, 41-55. https://doi.org/10.1016/j.cpr.2018.05.008

Goldberg, L. R. (1992). The development of markers for the Big-Five factor structure. Psychological Assessment, 4(1), 26-42. https://doi.org/10.1037/1040-3590.4.1.26

Green, P., \& MacLeod, C. J. (2016). SIMR: an R package for power analysis of generalized linear mixed models by simulation. Methods in Ecology and Evolution, 7(4), 493-498. https://doi.org/10.1111/2041-210X.12504

Hart, D., Atkins, R., Fegley, S., Robins, R. W., \& Tracy, J. L. (2003). Personality and development in childhood: A person-centered approach. Monographs of the Society for Research in Child Development, 68(1), i-iii+v+vii+1-122.

Hart, D., Hofmann, V., Edelstein, W., \& Keller, M. (1997). The relation of childhood personality types to adolescent behavior and development: A longitudinal study of Icelandic children. Developmental Psychology, 33(2), 195-205. https://doi.org/10.1037/0012-1649.33.2.195

Henning, G., Hansson, I., Berg, A. I., Lindwall, M., \& Johansson, B. (2017). The role of personality for subjective well-being in the retirement transition-Comparing variable-and person-oriented models. Personality and Individual Differences, 116, 385-392. https://doi.org/10.1016/j.paid.2017.05.017

Herzberg, P. Y., \& Roth, M. (2006). Beyond resilients, undercontrollers, and overcontrollers? An extension of personality prototype research. European fournal of Personality, 20(1), 5-28. https://doi.org/10.1002/per.557

Infurna, F. J., \& Luthar, S. S. (2016). Resilience to major life stressors is not as common as thought. Perspectives on Psychological Science, 11(2), 175-194. https://doi.org/10.1177/1745691615621271

Infurna, F. J., \& Luthar, S. S. (2017). The multidimensional nature of resilience to spousal loss. Journal of Personality and Social Psychology, 112(6), 926-947. https://doi.org/10.1037/pspp0000095

Isler, L., Liu, J. H., Sibley, C. G., \& Fletcher, G. J. (2016). Self-regulation and personality profiles: Empirical development, longitudinal stability and predictive ability. European fournal of Personality, 30(3), 274-287. https://doi.org/10.1002/per.2054

Kim, T. J., \& von Dem Knesebeck, O. (2016). Perceived job insecurity, unemployment and depressive symptoms: A systematic review and meta-analysis of prospective observational studies. International Archives of Occupational and Environmental Health, 89(4), 561-573. https://doi.org/10.1007/s00420-015-1107-1 
Klimstra, T. A., Hale, W. W., III, Raaijmakers, Q. A., Branje, S. J., \& Meeus, W. H. (2010). A developmental typology of adolescent personality. European fournal of Personality, 24(4), 309-323. https://doi.org/10.1002/per.744

Leikas, S., \& Salmela-Aro, K. (2014). Personality types during transition to young adulthood: How are they related to life situation and well-being? fournal of Adolescence, 37(5), 753-762. https://doi.org/10.1016/j.adolescence.2014.01.003

Lucas, R. E. (2005). Time does not heal all wounds: A longitudinal study of reaction and adaptation to divorce. Psychological Science, 16(12), 945-950. https://doi.org/10.1111/j.1467-9280.2005.01642.x

Lucas, R. E., Clark, A. E., Georgellis, Y., \& Diener, E. (2004). Unemployment alters the set point for life satisfaction. Psychological Science, 15(1), 8-13. https://doi.org/10.1111/j.0963-7214.2004.01501002.x

Luciano, E. C., \& Orth, U. (2017). Transitions in romantic relationships and development of selfesteem. Journal of Personality and Social Psychology, 112(2), 307-328. https://doi.org/10.1037/pspp0000109

Lugtig, P. (2014). Panel attrition: separating stayers, fast attriters, gradual attriters, and lurkers. Sociological Methods \& Research, 43(4), 699-723. https://doi.org/10.1177/0049124113520305

Luhmann, M., Fassbender, I., Alcock, M., \& Haehner, P. (2020). A dimensional taxonomy of perceived characteristics of major life events. Journal of Personality and Social Psychology; Advance online publication. https://doi.org/10.1037/pspp0000291

Luhmann, M., Hofmann, W., Eid, M., \& Lucas, R. E. (2012). Subjective well-being and adaptation to life events: A meta-analysis. Journal of Personality and Social Psychology, 102(3), 592-615. https://doi.org/10.1037/a0025948

Luthar, S. S., Cicchetti, D., \& Becker, B. (2000). The construct of resilience: A critical evaluation and guidelines for future work. Child Development, 71(3), 543-562. https://doi.org/10.1111/1467-8624.00164

Meeus, W., Van de Schoot, R., Klimstra, T., \& Branje, S. (2011). Personality types in adolescence: Change and stability and links with adjustment and relationships: A five-wave longitudinal study. Developmental Psychology, 47(4), 1181-1195. https://doi.org/10.1037/a0023816

Newman, D. L., Caspi, A., Moffitt, T. E., \& Silva, P. A. (1997). Antecedents of adult interpersonal functioning: Effects of individual differences in age 3 temperament. Developmental Psychology, 33(2), 206-217. https://doi.org/10.1037/0012-1649.33.2.206

Nylund, K., Asparouhov, T., \& Muthén, B. (2007). Deciding on the number of classes in latent class analysis and growth mixture modeling: A Monte Carlo simulation study. Structural Equation Modeling, 14(4), 535-569. https://doi.org/10.1080/10705510701575396

Pilarska, A. (2018). Big-Five personality and aspects of the self-concept: Variable-and personcentered approaches. Personality and Individual Differences, 127, 107-113.

https://doi.org/10.1016/j.paid.2018.01.049 
Recksiedler, C., \& Stawski, R. S. (2019). Marital transitions and depressive symptoms among older adults: Examining educational differences. Gerontology, 65, 407-418. https://doi.org/10.1159/000493681

Robins, R. W., John, O. P., Caspi, A., Moffitt, T. E., \& Stouthamer-Loeber, M. (1996). Resilient, overcontrolled, and undercontrolled boys: Three replicable personality types. Fournal of Personality and Social Psychology, 70(1), 157-171. https://doi.org/10.1037/0022-3514.70.1.157

Scherpenzeel, A. C. (2011). Data collection in a probability-based internet panel: How the LISS panel was built and how it can be used. Bulletin of Sociological Methodology. Bulletin de Methodologie Sociologique, 109, 56-61. https://doi.org/10.1177/0759106310387713

Scherpenzeel, A. C., \& Das, M. (2011). „True” longitudinal and probability-based internet panels: Evidence from the Netherlands. In M. Das, P. Ester, \& L. Kaczmirek (Eds.), Social and behavioral research and the internet: Advances in applied methods and research strategies (pp. 77-104). Taylor \& Francis.

Scholte, R. H., van Lieshout, C. F., de Wit, C. A., \& van Aken, M. A. (2005). Adolescent personality types and subtypes and their psychosocial adjustment. Merrill-Palmer Quarterly, 51, 258-286. https://doi.org/10.1353/mpq.2005.0019

Schwaba, T., \& Bleidorn, W. (2019). Personality trait development across the transition to retirement. Fournal of Personality and Social Psychology, 116(4), 651-665. https://doi.org/10.1037/pspp0000179

Schwaba, T., Luhmann, M., Denissen, J. J., Chung, J. M., \& Bleidorn, W. (2018). Openness to experience and culture-openness transactions across the lifespan. fournal of Personality and Social Psychology, 115(1), 118-136. https://doi.org/10.1037/pspp0000150

Schwarz, G. (1978). Estimating the dimension of a model. Annals of Statistics, 6(2), 461-464. https://doi.org/10.1214/aos/1176344136

Siflinger, B. (2017). The effect of widowhood on mental health - An analysis of anticipation patterns surrounding the death of a spouse. Health Economics, 26(12), 1505-1523.

https://doi.org/10.1002/hec.3443

Specht, J., Egloff, B., \& Schmukle, S. C. (2011). Stability and change of personality across the life course: The impact of age and major life events on mean-level and rank-order stability of the Big Five. Journal of Personality and Social Psychology, 101(4), 862-882. https://doi.org/10.1037/a0024950

Spinhoven, P., Elzinga, B. M., Van Hemert, A. M., de Rooij, M., \& Penninx, B. W. (2016). Childhood maltreatment, maladaptive personality types and level and course of psychological distress: A six-year longitudinal study. Journal of Affective Disorders, 191, 100-108.

https://doi.org/10.1016/j.jad.2015.11.036

Steca, P., Alessandri, G., \& Caprara, G. V. (2010). The utility of a well-known personality typology in studying successful aging: Resilients, undercontrollers, and overcontrollers in old age. Personality and Individual Differences, 48(4), 442-446. https://doi.org/10.1016/j.paid.2009.11.016 Vable, A. M., Subramanian, S., Rist, P. M., \& Glymour, M. M. (2015). Does the "widowhood effect" precede spousal bereavement? Results from a nationally representative sample of older adults. 
The American fournal of Geriatric Psychiatry, 23(3), 283-292.

https://doi.org/10.1016/j.jagp.2014.05.004

van Aken, M. A., \& Semon Dubas, J. (2004). Personality type, social relationships, and problem behaviour in adolescence. European fournal of Developmental Psychology, 1(4), 331-348. https://doi.org/10.1080/17405620444000166

Van der Laan, J. (2009). Representativity of the LISS Panel (Discussion paper No. 09041). https://citeseerx.ist.psu.edu/viewdoc/download?doi=10.1.1.680.9416\&rep=rep1\&type=pdf Vermunt, J. K., \& Magidson, J. (2013). Latent GOLD 5.0 Upgrade Manual. Statistical Innovations.

Ware, J. E., Jr., \& Sherbourne, C. (1992). The MOS Short-Form Health Survey (SF-36): I. Conceptual framework and item selection. Medical Care, 30(6), 473-483.

https://doi.org/10.1097/00005650-199206000-00002

Westerhof, G. J., \& Keyes, C. L. (2010). Mental illness and mental health: The two continua model across the lifespan. Fournal of Adult Development, 17(2), 110-119. https://doi.org/10.1007/s10804-009-9082-y

Yu, R., Branje, S., Keijsers, L., \& Meeus, W. H. (2014a). Personality effects on romantic relationship quality through friendship quality: A ten-year longitudinal study in youths. PLOS ONE, 9, Article e102078. https://doi.org/10.1371/journal.pone.0102078

Yu, R., Branje, S. J., Keijsers, L., \& Meeus, W. H. (2014b). Personality types and development of adolescents' conflict with friends. European fournal of Personality, 28(2), 156-167. https://doi.org/10.1002/per.1913

\section{eapp}

Personality Science (PS) is an official journal of the European Association of Personality Psychology (EAPP).
(4) leibniz-psychology.org

PsychOpen GOLD is a publishing service by Leibniz Institute for Psychology (ZPID), Germany. 